\title{
Association between the CYP1A2 rs762551 Polymorphism and Bladder Cancer Susceptibility: a Meta-Analysis Based on Case-Control Studies
}

\author{
Yong Zeng ${ }^{1}$, Hua-Yong Jiang ${ }^{2}$, Li Wei ${ }^{3}$, Wei-Dong $\mathrm{Xu}^{2}$, Ya-Jie Wang ${ }^{4}$, Ya-Di \\ Wang $^{2}$, Chuan Liu ${ }^{4 *}$
}

\begin{abstract}
Background: Previous studies evaluated associations between the CYP1A2 rs762551 polymorphism and bladder cancer risk. However, the results were inconsistent. We therefore performed a meta-analysis of the published case-control studies to assess in detail the association between CYP1A2 rs762551 polymorphism and bladder cancer risk. Materials and Methods: PubMed, Embase and Web of Science were searched to identify relevant studies and the pooled odds ratio $(\mathrm{OR})$ and $95 \%$ confidence interval $(95 \% \mathrm{CI})$ were calculated. Results: A total of seven articles including 3,013 cases and 2,771 controls were finally included. Overall, a significant association was found between the CYP1A2 rs762551 polymorphism and bladder cancer susceptibility for CC vs $\mathrm{AA}(\mathrm{OR}=0.82,95 \% \mathrm{CI}=0.69 \sim 0.99)$, but no significant associations were found for the other three models (AC vs AA: $O R=0.91,95 \% \mathrm{CI}=0.81 \sim 1.02$; the dominant model: $\mathrm{OR}=0.90,95 \% \mathrm{CI}=0.80 \sim 1.00$; the recessive model: $\mathrm{OR}=0.84,95 \% \mathrm{CI}=\mathbf{0 . 7 2} \mathbf{1 . 0 0}$ ). In the subgroup analysis by ethnicity, we detected significant associations between the CYP1A2 rs762551 polymorphism and bladder cancer susceptibility for GA vs GG (OR = 0.78, 95\% $\mathrm{CI}=0.64 \sim 0.96)$ and for the recessive model $(\mathrm{OR}=0.80,95 \% \mathrm{CI}=0.66 \sim 0.96)$ in Caucasians, but not for Asians. Conclusions: The results from the meta-analysis suggested that the CYP1A2 rs762551 polymorphism is a protective factor for bladder cancer, especially in Caucasians.
\end{abstract}

Keywords: Meta-analysis - CYP1A2 rs762551 - bladder cancer - susceptibility - ethnicity

Asian Pac J Cancer Prev, 16 (16), 7249-7254

\section{Introduction}

Bladder cancer (BC) is the most common malignant disease with poor prognosis and considered a significant public health threat all over the world (Siegel et al., 2014). As a complex and multifactorial process, the bladder carcinogenesis is still not fully understood. Epidemiological research has suggested that bladder cancer is a multifactorial disease with a wide variety of disease-producing factors such as environmental, genetic, and some new emerging risk factors (Kiemeney et al., 2009; Kiltie, 2010). Tobacco smoking, exposure to industrial aromatic amines, and intake of carcinogenic drugs such as phenacetin, chlornaphazine, and cyclophosphamide are the most important risk factors for bladder cancer (Volanis et al., 2010). It has also been documented that expression of gene and protein was affected by a single nucleotide polymorphisms (SNPs) located within the promoter or other regulatory regions of the gene, thus contributing to an individual's susceptibility to bladder cancer.

In recent years, several common low-penetrant genes have been identified as potential bladder cancer susceptibility genes. Cytochrome P4501A2 (CYP1A2) is a key phase I enzyme necessary to activate the major recognized bladder carcinogens, i.e., aromatic amines (AAs) and polycyclic aromatic hydrocarbons (PAHs), which require metabolic activation before exerting their damaging effects (Caporaso et al., 1991). Increased CYP1A2 activity was associated to the inducibility of CYP1A2 (Butler et al., 1992; Pavanello et al., 2002) by a number of environmental factors, such as AAs, PAHs, heterocyclic amines, nitrosamines, whose exposure occurs via tobacco smoking, occupational exposure, diet (Sesardic et al., 1988; Nordmark et al., 1999; Chung et al., 2000). Genetic and epigenetic changes may alter the activity of $C Y P 1 A 2$ and its downstream signaling, and may also affect an individual's susceptibility to human cancers at various sites (Rodriguez-Antona and IngelmanSundberg, 2006). Therefore, it is reasonable that CYP1A2 may play an important role in the etiology cancer risk. More than 17 SNPs were identified in the CYP1A2 gene but few of them have been reported to affect the activity of

${ }^{I}$ Department of Cardiothoracic Surgery, Shaoxing People's Hospital, Shaoxing Hospital of Zhejiang University, Shaoxing, Zhejiang Province, ${ }^{2}$ Department of Radiation Oncology, Beijing Military General Hospital, Dongchen, Beijing, ${ }^{3}$ Department of Oncology, the 401 hospital of PLA, Qingdao, Shangdong, ${ }^{4}$ Department of Oncology, Changhai Hospital, Second Military Medical University, Shanghai, China*For correspondence: chuanliu2005@163.com 
Yong Zeng et al

CYP1A2 (Sachse et al., 2003; Li et al., 2006; Sangrajrang et al., 2009; Qiu et al., 2010). The CYP1A2 rs762551 polymorphism $(C Y P 1 A 2 * 1 \mathrm{~F},-164 \mathrm{~A} / \mathrm{C}, \mathrm{C} \rightarrow \mathrm{A})$ is one of the most common studied variants, the polymorphism was in intron 1 of the CYPIA2 gene at position 734 downstream of the first transcribed nucleotide (Sachse et al., 1999).

To date, a number of studies have reported the association between $C Y P 1 A 2$ rs 762551 polymorphism and bladder cancer risk. However, previous studies on the association between $C Y P 1 A 2$ rs 762551 polymorphism and risk of bladder cancer reported inconsistent findings, partially because of the possible small effect of the polymorphism on bladder cancer risk and the relatively small sample size in each of published studies. Therefore, we performed this meta-analysis to derive a more precise estimation of this association.

\section{Materials and Methods}

\section{Search strategy}

PubMed, Embase and Web of Science were searched to identify relevant studies on the association between CYP1A2 rs762551 polymorphism and risk of bladder cancer, with a time limit of Mar 23, 2015. The following search terms was used: "cytochrome P-450 1A2", "CYP1A2", "polymorphism" and "bladder cancer". There was no language limitation in the search of databases. In addition, we checked the references of relevant reviews and eligible articles to find other possible studies.

\section{Selection criteria}

To be included in the analysis, candidate studies had to meet the following criteria: (1) Case-control study focused on the relationship between the CYP1A2 rs762551 polymorphism and bladder cancer risk, (2) All patients met the diagnostic criteria for glioma, (3) Sufficient original data for calculating odds ratios (ORs) with corresponding $95 \%$ confidence intervals $(95 \% \mathrm{CIs})$.

\section{Data extraction}

Information was carefully extracted from all eligible publications independently by two of the authors according to the inclusion criteria listed above. Disagreement was resolved by discussion between the other authors. For each study, the following characteristics were collected: the first author's name, country or region, year of publication, study design, method of genotyping, total numbers of cases and controls, and numbers of cases and controls who harbored the CYP1A2 rs762551 polymorphism. The ethnicity descents were categorized as Caucasians and Asians.

Quality assessment

The Newcastle-Ottawa Scale (NOS) criteria were used to assess the quality of each included study (Stang, 2010). The NOS criteria use a "star" rating system to judge methodological quality based on three aspects of a study: selection, comparability, and exposure. Scores range from 0 stars (worst) to 9 stars (best), with scores of 5 or higher indicating a moderate-high methodological quality.

\section{Statistical methods}

HWE software (http://linkage.rockefeller.edu/ott/ linkutil.htm) was used for a Hardy-Weinberg equilibrium (HWE) test. The chi-squared goodness-of-fit test was used to test deviation from Hardy-Weinberg equilibrium (HWE; $p<0.05$ was considered significant). Crude ORs with $95 \%$ CIs were used to assess the strength of association between CYP1A2 rs762551 polymorphism and bladder cancer risk. Heterogeneity assumptions were assessed by chi-square-based Q-test (Cochran, 1954). A $\mathrm{P}$ value greater than 0.10 for the Q-test indicated a lack of heterogeneity among the studies. Thus, the pooled OR estimate of each study was calculated using the fixedeffects model (the Mantel-Haenszel method) (Mantel and Haenszel, 1959); otherwise, the random effects model (the DerSimonian and Laird method) was used (DerSimonian and Laird, 1986). Meanwhile, we measured the effect of heterogeneity by another measure, $\mathrm{I}^{2}=100 \% \times(\mathrm{Q}-\mathrm{df}) / \mathrm{Q}$ (Higgins and Thompson, 2002). Venice criteria (Ioannidis et al., 2008) for the $\mathrm{I}^{2}$ test included: " $\mathrm{I}^{2}<25 \%$ represents no heterogeneity, $\mathrm{I}^{2}=25-50 \%$ represents moderate heterogeneity, $\mathrm{I}^{2}=50-75 \%$ represents large heterogeneity and $\mathrm{I}^{2}>75 \%$ represents extreme heterogeneity". The pooled ORs were performed for co-dominant model (CC vs AA and $\mathrm{CA} v s \mathrm{AA})$, the dominant model (CC+CA vs AA), and the recessive model (CC vs $\mathrm{CA}+\mathrm{AA})$, respectively. Subgroup analyses were done by ethnicity. An estimate of potential publication bias was carried out by the funnel plot, in which the standard error of $\log (\mathrm{OR})$ of each study was plotted against its $\log (\mathrm{OR})$. Publication bias may be absent if the plot resembles a symmetrical inverted funnel in which smaller, less precise, and more numerous studies have increasingly large variation in the estimates of their effect size (Sutton et al., 2000). Publication bias was further investigated using Begg's funnel plot and Egger's regression test (Begg and Mazumdar, 1994; Egger et al., 1997) $(p<0.05$ was considered to be statistically significant). All statistical analyses were performed using STATA statistical software (version 10.0). Two-sided $p$ values less than 0.05 were considered statistically significant.

\section{Results}

\section{Study characteristics}

The characteristics of included studies: A total of six articles were collected according to the search criteria (Tsukino et al., 2004; Figueroa et al., 2008; Altayli et al., 2009; Villanueva et al., 2009; Pavanello et al., 2010; Cui et al., 2013). One publication (Villanueva et al., 2009) contained two case-control data was considered to two separate studies, therefore, seven articles including 3,013 cases and 2,771 controls were used for this meta-analysis. The characteristics of the included studies are shown in Table 1 . The controls were primarily healthy population. All of the cases were pathologically confirmed. In terms of ethnicity, there were three groups of Asians (Tsukino et al., 2004; Altayli et al., 2009; Cui et al., 2013), four groups of Caucasions (Figueroa et al., 2008; Villanueva et al., 2009; Pavanello et al., 2010). Studies with control 
The CYP1A2 rs762551 Polymorphism and Bladder Cancer Susceptibility: A Meta-Analysis Based on Case-Control Studies Table 1. Characteristics of Studies of the CYP1A2 rs762551 Polymorphism and Bladder Cancer Susceptibility

\begin{tabular}{|c|c|c|c|c|c|c|c|c|c|c|c|c|}
\hline Study & \multirow{2}{*}{ Country } & \multirow{2}{*}{ Gene test } & \multirow{2}{*}{ Source } & \multirow{2}{*}{$\begin{array}{l}\text { No. of } \\
\text { Case/ } \\
\text { Control }\end{array}$} & \multicolumn{3}{|c|}{ Case } & \multicolumn{3}{|c|}{ Control } & \multirow{2}{*}{ HWE } & \multirow{2}{*}{ NOS } \\
\hline rs762551 & & & & & AA & $\mathrm{CA}$ & $\mathrm{CC}$ & AA & $\mathrm{CA}$ & $\mathrm{CC}$ & & \\
\hline Cui X 2012 & Asian & PCR-RFLP & $\mathrm{HB}$ & $282 / 257$ & 169 & 94 & 19 & 147 & 99 & 11 & 0.26 & 8 \\
\hline Pavanello S 2010 & Caucasion & PCR-RFLP & $\mathrm{HB}$ & $155 / 148$ & \multicolumn{2}{|c|}{145} & 10 & \multicolumn{2}{|c|}{132} & 16 & - & 7 \\
\hline Altayli E 2009 & Asian & TaqMan & $\mathrm{HB}$ & $135 / 128$ & 44 & 64 & 27 & 31 & 64 & 33 & 1 & 8 \\
\hline Villanueva CM 2009 & Caucasion & TaqMan & $\mathrm{HB}$ & $890 / 804$ & 397 & 395 & 98 & 332 & 361 & 111 & 0.42 & 8 \\
\hline Villanueva CM 2009 & Caucasion & TaqMan & $\mathrm{HB}$ & $144 / 107$ & 61 & 68 & 15 & 48 & 51 & 8 & 0.26 & 8 \\
\hline Figueroa JD 2008 & Caucasion & TaqMan & $\mathrm{HB}$ & $1101 / 1021$ & 482 & 499 & 120 & 422 & 464 & 135 & 0.68 & 9 \\
\hline Tsukino H 2004 & Asian & PCR-RFLP & PB & $306 / 306$ & 183 & 104 & 19 & 179 & 113 & 14 & 0.47 & 8 \\
\hline
\end{tabular}

*HB: hospital based; PB: population based; HWE: Hardy-Weinberg equilibrium; PCR: Polymerase chain reaction; RFLP: Restriction fragment length polymorphism; NOS: Newcastle-Ottawa Scale

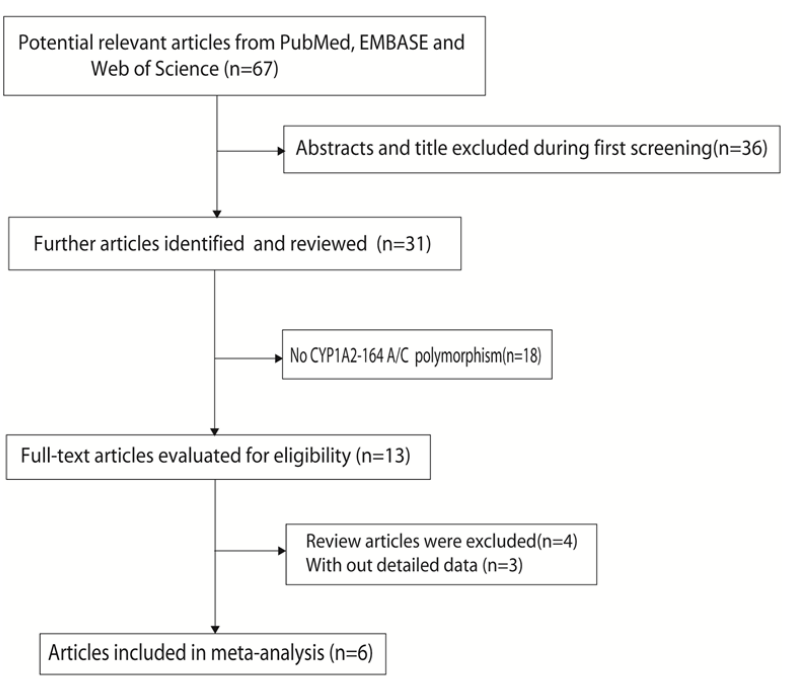

Figure 1. Study Selection Process for the Meta-analysis

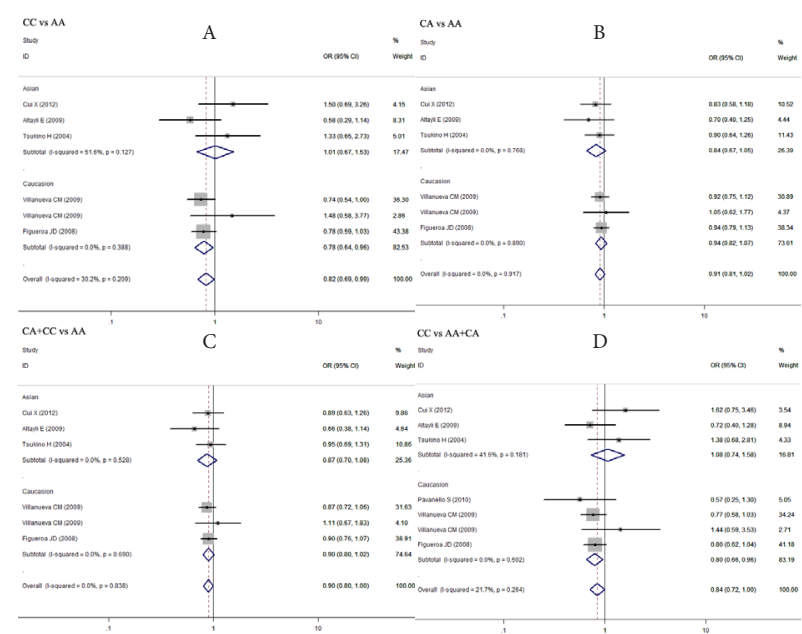

Figure 2. Odds Ratios (ORs) for Associations between the CYP1A2 rs762551 Polymorphism and Bladder Susceptibility

not in Hardy-Weinberg equilibrium (HWE) were also considered for meta-analysis, but they were excluded in the sensitivity analysis (Minelli et al., 2008).

\section{Quantitative synthesis}

The main results of our meta-analysis under four distinct genetic models were listed in Table 2 and Table

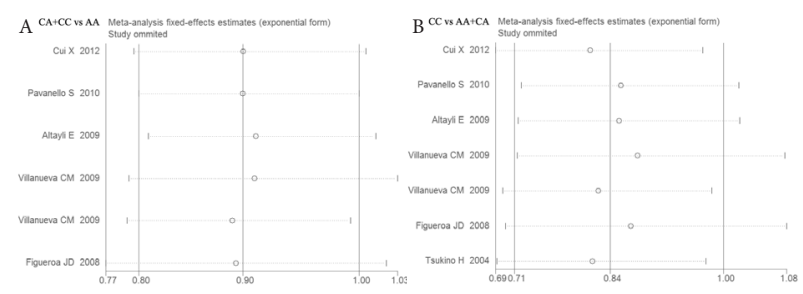

Figure 3. Sensitivity Analysis Using the One-Study Remove Approach in the Dominant Model and the Recessive Model

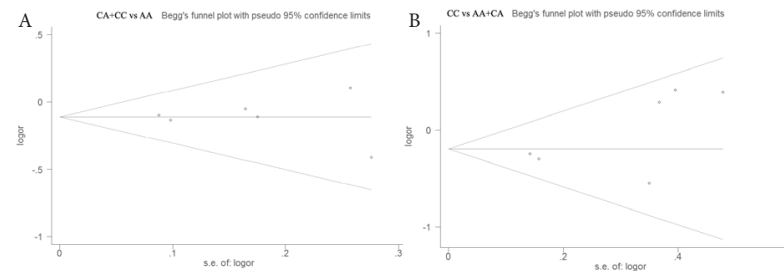

Figure 4. Publication Bias in Studies of the Relation between the CYP1A2 rs762551 Polymorphism and Bladder Susceptibility in the Dominant Model and the Recessive Model. A funnel plot with pseudo-95\% confidence limits (dashed lines) was used

3. Overall, a significant association was found between the CYP1A2 rs762551 polymorphism and bladder cancer susceptibility for CC vs AA (OR=0.82, 95\% $\mathrm{CI}=0.69 \sim 0.99, p=0.21$ for heterogeneity, Figure $2 \mathrm{~A}$ ), but no significant association was found for the other three models (Ac vs AA: OR=0.91, 95\% CI=0.81 1.02, $p=0.92$ for heterogeneity, Figure 2B; The dominant model: $\mathrm{OR}=$ $0.90,95 \% \mathrm{CI}=0.80 \sim 1.00, p=0.84$ for heterogeneity, Figure $2 \mathrm{C}$; the recessive model: $\mathrm{OR}=0.84,95 \% \mathrm{CI}=0.72 \sim 1.00$, $p=0.26$ for heterogeneity, Figure 2D).

In the subgroup analysis by ethnicity, in Caucasian population, we detected significant associations between the CYP1A2 rs762551 polymorphism and bladder cancer susceptibility for GA vs GG $(\mathrm{OR}=0.78,95 \%$ $\mathrm{CI}=0.64 \sim 0.96, p=0.39$ for heterogeneity) and for the recessive model $(\mathrm{OR}=0.80,95 \% \mathrm{CI}=0.66 \sim 0.96, p=0.50$ for heterogeneity). But for Asian population, no significant association was found.

\section{Heterogeneity and sensitivity analysis}

There were no significant heterogeneities for the overall analysis and the subgroup analysis under all four 
Table 2. Summary Odds Ratios Relations between the CYP1A2 rs762551 Polymorphism and Bladder Cancer Susceptibility

\begin{tabular}{|c|c|c|c|c|c|c|c|c|c|c|c|}
\hline \multirow{2}{*}{ Polymorphism } & \multirow{2}{*}{$\begin{array}{c}\text { Genetic } \\
\text { model }\end{array}$} & \multirow{2}{*}{$\begin{array}{c}\text { Genetic } \\
\text { type }\end{array}$} & \multicolumn{3}{|c|}{ Heterogeneity test } & \multirow{2}{*}{ OR $(95 \% \mathrm{CI})$} & \multirow{2}{*}{ P1 } & \multicolumn{2}{|c|}{ Begg's test } & \multicolumn{2}{|c|}{ Egger's test } \\
\hline & & & Q & $\mathrm{I}^{2}(\%)$ & $\mathrm{PH}$ & & & $\mathrm{Z}$ & $\mathrm{P} 2$ & $\mathrm{t}$ & P3 \\
\hline \multirow{4}{*}{ rs762551 } & \multirow{2}{*}{$\begin{array}{c}\text { Codominant } \\
\text { model }\end{array}$} & $\begin{array}{c}\mathrm{CC} \text { vs } \\
\mathrm{AA}\end{array}$ & 7.17 & $30.20 \%$ & 0.21 & $0.82(0.69 \sim 0.99)$ & 0.04 & 0.75 & 0.45 & 1.79 & 0.15 \\
\hline & & $\begin{array}{c}\text { CA vs } \\
\text { AA }\end{array}$ & 1.47 & $0.00 \%$ & 0.92 & $0.91(0.81 \sim 1.02)$ & 0.11 & 1.13 & 0.26 & -1.04 & 0.36 \\
\hline & $\begin{array}{c}\text { Dominant } \\
\text { model }\end{array}$ & $\begin{array}{c}\mathrm{CA}+\mathrm{CC} \\
\mathrm{vs} \mathrm{AA}\end{array}$ & 2.08 & $0.00 \%$ & 0.84 & $0.90(0.80 \sim 1.00)$ & 0.04 & 0 & 1 & -0.11 & 0.91 \\
\hline & $\begin{array}{c}\text { Recessive } \\
\text { model }\end{array}$ & $\begin{array}{c}\mathrm{CC} \text { vs } \\
\mathrm{CA}+\mathrm{AA}\end{array}$ & 7.66 & $21.70 \%$ & 0.26 & $0.84(0.72 \sim 1.00)$ & 0.05 & 0.3 & 0.76 & 1.41 & 0.22 \\
\hline
\end{tabular}

*PH value for heterogeneity; P1 value for OR; P2 value for Begg's test; P3 value for Egger's test; OR: Odds ratio; CI: Confidence interval

Table 3. Main Results of Pooled Odds Ratios (OR) with Confidence Interval (CI) in the Meta-analysis by Ethnicity, and Gene Test

\begin{tabular}{|c|c|c|c|c|c|c|c|c|c|c|c|}
\hline & $\begin{array}{l}\text { morphism } \\
\operatorname{rroup}(\mathrm{N})\end{array}$ & Genetic type & \multicolumn{3}{|c|}{ Heterogeneity test } & \multirow{2}{*}{ OR $(95 \% \mathrm{CI})$} & \multirow{2}{*}{$\mathrm{P} 1$} & \multicolumn{2}{|c|}{ Begg's test } & \multicolumn{2}{|c|}{ Egger's test } \\
\hline \multicolumn{3}{|c|}{ rs762551 } & Q & $\mathrm{I}^{2}(\%)$ & $\mathrm{PH}$ & & & $\mathrm{Z}$ & $\mathrm{P} 2$ & $\mathrm{t}$ & P3 \\
\hline \multirow{4}{*}{\multicolumn{2}{|c|}{$\operatorname{Asian}(3)$}} & $\mathrm{CC}$ vs AA & 4.13 & $51.60 \%$ & 0.12 & $1.01(0.67 \sim 1.53)$ & 0.96 & 1.04 & 0.3 & 20.38 & 0.33 \\
\hline & & CA vs AA & 0.53 & $0.00 \%$ & 0.77 & $0.84(0.67 \sim 1.05)$ & 0.12 & 1.04 & 0.3 & -1.84 & 0.24 \\
\hline & & $\mathrm{CA}+\mathrm{CC}$ vs $\mathrm{AA}$ & 1.28 & $0.00 \%$ & 0.53 & $0.87(0.71 \sim 1.08)$ & 0.53 & 1.04 & 0.3 & -3.15 & 0.06 \\
\hline & & $\mathrm{CC}$ vs $\mathrm{CA}+\mathrm{AA}$ & 3.42 & $41.60 \%$ & 0.18 & $1.08(0.74 \sim 1.58)$ & 0.7 & 1.04 & 0.3 & 8.92 & 0.06 \\
\hline \multirow{4}{*}{$\operatorname{Rac}$} & \multirow{4}{*}{ Caucasian(4) } & $\mathrm{CC}$ vs AA & 1.9 & $0.00 \%$ & 0.39 & $0.78(0.64 \sim 0.96)$ & 0.02 & 0 & 1 & 1.95 & 0.18 \\
\hline & & $\mathrm{CA}$ vs AA & 0.23 & $0.00 \%$ & 0.89 & $0.94(0.79 \sim 1.13)$ & 0.33 & 0 & 1 & 0.66 & 0.36 \\
\hline & & $\mathrm{CA}+\mathrm{CC}$ vs $\mathrm{AA}$ & 0.74 & $0.00 \%$ & 0.69 & $0.90(0.80 \sim 1.02)$ & 0.69 & 0 & 1 & 1.24 & 0.28 \\
\hline & & $\mathrm{CC}$ vs $\mathrm{CA}+\mathrm{AA}$ & 2.36 & $21.70 \%$ & 0.5 & $0.80(0.66 \sim 0.96)$ & 0.02 & -0.34 & 1 & 0.41 & 0.75 \\
\hline
\end{tabular}

* $\mathrm{N}$ for numbers of studies; PH value for heterogeneity; P1 value for OR; P2 value for Begg's test; P3 value for Egger's test; OR: Odds ratio; CI: Confidence interval

genetic models by ethnicity, so the results were assessed under fixed effects model. Sensitivity analysis was performed to reflect the impact of the individual study to the summarized ORs by removing one study each time involved in the meta-analysis. Such a leave-oneout sensitivity analysis indicated that no single study influenced the pooled ORs qualitatively in the dominant model (Figure 3A), the recessive model (Figure 3B) and other models (not showed).

\section{Publication bias}

In this meta-analysis, we used funnel plots, Begg's test and Egger's linear regression method to evaluate the publication bias. The funnel plots for publication bias showed symmetry for the dominant model and the recessive model (Figure 4A, Figure 4B). Meanwhile, results of Begg's test and Eggers's linear regression method indicated that there was no obvious publication bias $(\mathrm{P}>0.05$, Table 2$)$.

\section{Discussion}

CYP1A2 is a key factor in the metabolic activity of carcinogenic aromatic and heterocyclic amines, the inhibition activity of this enzyme may represent a logical strategy for preventing the development of human cancers induced by the aromatic and heterocyclic amines (Miranda et al., 2000). Considering the important roles of CYP1A2, genetic polymorphisms in the CYPIA2 gene may have some effects on the development of cancer (Bozina et al., 2009). The association between the CYP1A2 rs762551 polymorphism and bladder cancer susceptibility had been studied extensively but the results had been inconsistent. In order to resolve this conflict, we thus performed a metaanalysis of the published case-control studies to assess the association between $C Y P 1 A 2$ rs762551 polymorphism and risk of bladder cancer. The present meta-analysis is the first meta-analysis on the association between $C Y P 1 A 2$ rs 762551 polymorphism and risk of bladder cancer up to now.

In the present meta-analysis, seven studies with a total of 3,013 cases and 2,771 controls were finally included, the results showed that $C Y P 1 A 2$ rs762551 polymorphism was a protective factor for bladder cancer risk, the subgroup analysis based on ethnicity further suggested that CYP1A2 rs 762551 polymorphism was a protective factor for bladder cancer risk in Caucasian population, but not in Asian population. In addition, the leave-one-out analysis suggested that omitting each study had limited effect on the pooled estimates.

Cancer development was a complicated process involving many genes, different genetic backgrounds 
The CYP1A2 rs762551 Polymorphism and Bladder Cancer Susceptibility: A Meta-Analysis Based on Case-Control Studies

might contribute to the discrepancy. It was reported that C allele causing decreased activity of the encoded enzyme may lead to decreased metabolism of estradiol and $\mathrm{C}$ allele carriers might potentially increase the bladder cancer risk. Actually, it might be not uncommon that the epidemiology results were not coincident with the results of functional study. The influence of the $\mathrm{C}$ allele might be decreased by the presence of other unidentified causal genes involved in bladder cancer susceptibility.

Meta-analysis has been recognized as an effective method to solve a wide variety of clinical questions by summarizing and reviewing the previously published quantitative research. By using meta-analysis, a multitude of genetic polymorphisms have been associated with specific disease states. By using meta-analysis, XPD Lys751Gln (Xiong et al., 2014), CYP1A1 11599G>C (Wang et al., 2014), NQO1 Exon 6 (Mandal et al., 2014) polymorphisms have been proved associated with bladder cancer susceptibility.

Some limitations of this meta-analysis should be acknowledged. Firstly, the controls were not uniformly defined. Although most of the controls were selected mainly from healthy populations, some had benign disease. Therefore, non-differential misclassification bias was possible because these studies may have included the control groups who have different risks of developing bladder cancer.

Second, as a type of a retrospective study, a metaanalysis may encounter recalls or selection bias, possibly influencing the reliability of our study results. Third, the overall outcomes were based on unadjusted estimates, while a more precise evaluation should be adjusted by other co-variants including tobacco use, alcohol consumption, viral infection, and environment factors if individual data were available.

In conclusion, the current meta-analysis provides obvious evidence that CYP1A2 rs762551 polymorphism was a protective factor for bladder cancer susceptibility, especially in Caucasians. Large and well-designed epidemiological studies will be necessary to combine genetic factors together with other potential risk factor in order to validate the association between the CYP1A2 rs762551 polymorphism and bladder cancer susceptibility.

\section{Acknowledgements}

This study was supported by China Postdoctoral Science Foundation (No. 2014M562541), Natural Science Foundation of the People's Republic of China (No. $81102010 ; 81202096 ; 81372854)$. The fundershad no role in study design, data collection and analysis, decision to publish, or preparation of the manuscript.

\section{References}

Altayli E, Gunes S, Yilmaz AF, Goktas S, Bek Y (2009). CYP1A2, CYP2D6, GSTM1, GSTP1, and GSTT1 gene polymorphisms in patients with bladder cancer in a Turkish population. Int Urol Nephrol, 41, 259-66.

Begg CB, Mazumdar M (1994). Operating characteristics of a rank correlation test for publication bias. Biometrics, $\mathbf{5 0}$,
1088-101.

Bozina N, Bradamante V,Lovric M (2009). Genetic polymorphism of metabolic enzymes P450 (CYP) as a susceptibility factor for drug response, toxicity, and cancer risk. Arh Hig Rada Toksikol, 60, 217-42

Butler MA, Lang NP, Young JF, et al (1992). Determination of CYP1A2 and NAT2 phenotypes in human populations by analysis of caffeine urinary metabolites. Pharmacogenetics, 2, 116-27.

Chung WG, Kang JH, Park CS, Cho MH, Cha YN (2000). Effect of age and smoking on in vivo CYP1A2, flavin-containing monooxygenase, and xanthine oxidase activities in Koreans: determination by caffeine metabolism. Clin Pharmacol Ther, 67, 258-66.

Caporaso N, Landi MT, Vineis P(1991). Relevance of metabolic polymorphisms to human carcinogenesis: evaluation of epidemiologic evidence. Pharmacogenetics, 1, 4-19

Cochran WG (1954). The combination of estimates from different experiments. Biometrics, 10, 101-29.

Cui X, Lu X, Hiura M, et al (2013). Association of genotypes of carcinogen-metabolizing enzymes and smoking status with bladder cancer in a Japanese population. Environ Health Prev Med, 18, 136-42.

DerSimonian R, Laird N (1986). Meta-analysis in clinical trials. Control Clin Trials, 7, 177-88

Egger M, Davey Smith G, Schneider M, Minder C (1997). Bias in metaanalysis detected by a simple graphical test. $B M J$, 315, 629-34.

Figueroa JD, Malats N, Garcia-Closas M, et al (2008). Bladder cancer risk and genetic variation in AKR1C3 and other metabolizing genes. Carcinogenesis, 29, 1955-62.

Higgins JP, Thompson SG (2002). Quantifying heterogeneity in a meta-analysis. Stat Med, 21, 1539-58.

Ioannidis JP, Boffetta P, Little J, et al (2008). Assessment of cumulative evidence on genetic associations: interim guidelines. Int J Epidemiol, 37, 120-32.

Kiemeney LA, Grotenhuis AJ, Vermeulen SH, Wu X (2009). Genome-wide association studies in bladder cancer: first results and potential relevance. Curr Opin Urol, 19, 540-6.

Kiltie AE (2010). Common predisposition alleles for moderately common cancers: bladder cancer. Curr Opin Genet Dev, 20, 218-24.

Li D, Jiao L, Li Y, et al (2006). Polymorphisms of cytochrome $\mathrm{P} 4501 \mathrm{~A} 2$ and $\mathrm{N}$-acetyltransferase genes, smoking, and risk of pancreatic cancer. Carcinogenesis, 27, 103-11.

Mandal RK, Dubey S, Panda AK, Mittal RD (2014). Genetic variants of NQO1 gene increase bladder cancer risk in Indian population and meta-analysis. Tumour Biol, 35, 6415-23

Mantel N, Haenszel W (1959). Statistical aspects of the analysis of data from retrospective studies of disease. J Natl Cancer Inst, 22, 719-48

Minelli C, Thompson JR, Abrams KR, Thakkinstian A, Attia J (2008). How should we use information about HWE in the meta-analyses of genetic association studies? Int $J$ Epidemiol, 37, 136-46.

Miranda CL, Yang YH, Henderson MC, et al (2000). Prenylflavonoids from hops inhibit the metabolic activation of the carcinogenic heterocyclic amine 2-amino-3methylimidazo[4, 5-f]quinoline, mediated by cDNAexpressed human CYP1A2. Drug Metab Dispos, 28, 1297-302.

Nordmark A, Lundgren S, Cnattingius S, Rane A (1999). Dietary caffeine as a probe agent for assessment of cytochrome P4501A2 activity in random urine samples. Br J Clin Pharmacol, 47, 397-402.

Pavanello S, Simioli P, Lupi S, Gregorio P, Clonfero E (2002). Exposure levels and cytochrome P450 1A2 activity, but not 
$\mathrm{N}$-acetyltransferase, glutathione S-transferase (GST) M1 and $\mathrm{T} 1$, influence urinary mutagen excretion in smokers. Cancer Epidemiol Biomarkers Prev, 11, 998-1003.

Pavanello S, Mastrangelo G, Placidi D, et al (2010). CYP1A2 polymorphisms, occupational and environmental exposures and risk of bladder cancer. Eur J Epidemiol, 25, 491-500.

Qiu LX, Yao L, Mao C, Yu KD, Zhan P, et al (2010). Lack of association of CYP1A2-164 A/C polymorphism with breast cancer susceptibility: A meta-analysis involving 17,600 subjects. Breast Cancer Res Treat, 122, 521-5.

Rodriguez-Antona C, Ingelman-Sundberg M (2006). Cytochrome P450 pharmacogenetics and cancer. Oncogene, 25, 1679-91.

Sachse C, Bhambra U, Smith G, et al (2003). Colorectal Cancer Study Group. Polymorphisms in the cytochrome P450 CYP1A2 gene (CYP1A2) in colorectal cancer patients and controls: allele frequencies, linkage disequilibrium and influence on caffeine metabolism. Br J Clin Pharmacol, 55, 68-76.

Sachse C, Brockmoller J, Bauer S, Roots I (1999). Functional significance of a $\mathrm{C}>\mathrm{A}$ polymorphism in intron 1 of the cytochrome P450 CYP1A2 gene tested with caffeine. $\mathrm{Br} J$ Clin Pharmacol, 47, 445-9.

Sangrajrang S, Sato Y, Sakamoto H, et al (2009). Genetic polymorphisms of estrogen metabolizing enzyme and breast cancer risk in Thai women. Int J Cancer, 125, 837-43.

Sesardic D, Boobis AR, Edwards RJ, Davies DS (1988). A form of cytochrome $\mathrm{P} 450$ in man, orthologous to form $\mathrm{d}$ in the rat, catalyses the odeethylation of phenacetin and is inducible by cigarette smoking. Br J Clin Pharmacol, 26, 363-72.

Siegel R, Ma J, Zou Z, Jemal A (2014). Cancer statistics, 2014. CA Cancer J Clin, 64, 9-29.

Stang A (2010). Critical evaluation of the Newcastle-Ottawa scale for the assessment of the quality of nonrandomized studies inmeta-analyses. Eur J Epidemiol, 25, 603-5.

Sutton AJ, Duval SJ, Tweedie RL, Abrams KR, Jones DR (2000). Empirical assessment of effect of publication bias on metaanalysis. BMJ, 320, 1574-7.

Tsukino H, Kuroda Y, Nakao H, et al (2004). Cytochrome P450 (CYP) 1A2, sulfotransferase (SULT) 1A1, and $\mathrm{N}$-acetyltransferase (NAT) 2 polymorphisms and susceptibility to urothelial cancer.J Cancer Res Clin Oncol, 130, 99-106.

Villanueva CM, Silverman DT, Murta-Nascimento C, et al (2009). Coffee consumption, genetic susceptibility and bladder cancer risk. Cancer Causes Control, 20, 121-7.

Volanis D, Kadiyska T, Galanis A, et al (2010). Environmental factors and genetic susceptibility promote urinary bladder cancer. Toxicol Lett, 193, 131-7.

Wang Y, Kong CZ, Zhang Z, Yang CM, Li J (2014). Relationships between CYP1A1 genetic polymorphisms and bladder cancer risk: a meta-analysis. DNA Cell Biol, 33, 171-81.

Xiong T, Yang J, Wang H, et al (2014). The association between the Lys751Gln polymorphism in the XPD gene and the risk of bladder cancer. Mol Biol Rep, 41, 2629-34. 